\title{
Al-enabled Digital Transformation of Insurance Industry
}

\author{
Arun Chaubey \\ Insurance Domain Consultant \\ Zürich, Switzerland-8134
}

\author{
Sachchidanand Singh \\ Analytics \& Data Science SME \\ Pune, India-411045
}

\begin{abstract}
Insurance is a financial instrument used by insured, individuals and businesses, as a risk mitigation tool. It's a promise by the insurer that when an event occurs, a certain loss amount would be paid to the insured. Artificial Intelligence (AI) technologies like Machine Learning (ML) and Natural Language Processing (NLP) can handle processing of the large data volume of the insurance domain daily chores like automated claims, underwriting, chatbots more accurately and faster compared to the humans etc. Artificial intelligence (AI) is enhancing every step in the insurance value chain like instantly customizable life insurance and on-demand property coverage.
\end{abstract}

In this paper, we'd try to draw conclusions \& recommendations to perform successful digital transformations for an insurer based on our industry experiences of helping some of the world's largest insurers to go through that transformation journey successfully. We'd also explain the various avenues and touch points where latest technologies such as Artificial Intelligence (AI) can be used to create a market differentiator and value creator to the insurers.

\section{General Terms}

Artificial Intelligence (AI), Digital Technology, Machine Learning (ML), Natural Language Processing (NLP)

\section{Keywords}

Insurance, Underwriting, Automated Claims, Face Amount, Deductible, Co-payment, Reinsurance, Insurance Value Chain

\section{INTRODUCTION}

Digital Transformation requires not only technological changes but also cultural changes across the entire organization from top to bottom. Digital transformation in insurance companies is very different from any other sector as insurers have liability to manage \& continue serving their current customers while maintaining \& managing their data, business services, and promises to the market.

Digital transformation means integration of digital technology into all the areas of a business organization. In fact, its cultural change and organizations needs to continuously experiment, innovate newer ways and learn from their failures. Many repetitive work and daily chores like personalized recommendations, claims processing, underwriting can be automated, improved with the help of digital transformation and artificial intelligence (AI) technology ${ }^{[1],[2]}$.

Most insurers have terabytes of insurance data, but they are not sure how to leverage this data and quickly gain insights. With ever increasing customer demand and competitive landscape, insurance companies need to leverage data and knowledge in fast and effective manner to remain ahead and relevant in the changing market needs ${ }^{[3],[4],[5]}$.

\section{DIGITAL TRANSFORMATION IN INSURANCE INDUSTRY}

Insurance offerings can be generally classified into two lines of business - Life Insurance and General Insurance. The life insurance line focuses on the life $\&$ health aspects of the risks, while the General Insurance line of business focuses around property \& casualty aspects of the risks for individuals and businesses. Within the property \& casualty insurance line of business, risks can be further classified as personal and commercial class of businesses.

The commercial line of property \& casualty risks which is a segment serving insurance products to businesses - small, medium and large enterprises. The insurance offerings for commercial insureds could be different \& complex as compared with personal lines involved. In order to meet the businesses insurance needs, the product offering goes through several levels of changes and can be customized not only as a standalone product but also a complex mixed offering.

While products become complex, the business \& technological processes used to offer those products also become complex; and over the years, the insurer might have created several from manual to automated processes to meet their business's needs. And as the business grows, it becomes difficult to manage, maintain, and enhance those manual processes with regards to the changing market needs and market driven competition.

The processes which used to be differentiator and gave competitive advantages to the insurer; over the period, the very same processes become the challenges for the insurer as market evolves \& new entrants join the game. This eventually leads insurer to think about transforming itself to meet the growing market needs and challenges from competition - and the first answer that comes to the C-suite \& Board's mind is to go for a Digital Transformation.

By using digital technologies, insurance companies can benefit from cost competitiveness by paying based on the consumption rather than paying for software licenses, greater speed to the market and greater agility etc ${ }^{[6]}$.

\section{INSURANCE VALUE CHAIN}

In 1985, Michael Porter introduced concept of value chain to deliver maximum value to the end user for the least possible total cost. It expanded Leontief's input/output model by highlighting links between primary and support business activities. Michael Porter's value chain is basically a framework to develop an analytic structure which follows interdependent activities starting from raw idea to production and then into the hands of customer ${ }^{[7],[8]}$. 
Michael Porter's value chain model includes 05 primary activities- inbound logistics, operations, outbound logistics, marketing and sales, and service. And support activities are like procurement, human resources, technology development, and firm infrastructure. Porter's generic value chain model is adaptable to unique requirements and needs of every individual organizations for example, as the market evolvesdemands analysis and adjustment, growing market competitions, introduce new technology or ever-changing customer demands etc. And there are several benefits of value chain modeling like increased efficiency, cost reduction, competitive differentiation and higher-quality products at lower costs etc ${ }^{[9],[10]}$.

Insurance value chain is a set of activities that an insurance firm performs in order to deliver a valuable product or service for the market.
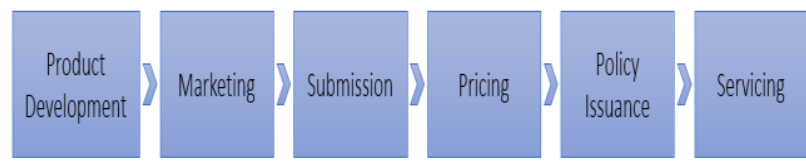

Fig 1. Insurance Value Chain

\section{ARTIFICIAL INTELLIGENCE (AI) IN INSURANCE INDUSTRY}

Most of the insurance companies process only certain percentage of large insurance data they have access to and kept under traditional databases. It means they are not able to unlock the advantage of valuable insights hidden in their unstructured data.

Artificial Intelligence (AI) and Robotic Process Automation (RPA) will become key differentiators in insurance domain. Collectively it will help organizations with better data processing and unique advantages of leveraging AIalgorithms for risk prediction and recommendations ${ }^{[11],[12]}$.

For example, insurance technology company's Lemonade's deploys Artificial Intelligence (AI) and behavioral economics as its core elements. Using AI, they are eliminating need of brokers and paperwork while behavioral economics capabilities are minimizing fraud and reducing overall time, effort and costs. Another insurance technology firm Tyche has deployed AI-infused claim likelihood model which helps in underwriting to increase profitability with accurate risk assessments.

With advancements in artificial intelligence (AI) and machine learning (ML), there is an increased demand of business chatbot in insurance domain to help automate policy servicing and claims managements by providing personalized customer experience and faster processing [13],[14]. For example, Lemonade's claims bot Jim does the assessments and rolls out property claims at faster rate.

The initial impact of artificial intelligence (AI) will be on automating underwriting, claims processes and taking over redundant tasks to improve efficiencies. The traditional insurers will have to move quickly to infuse artificial intelligence (AI) throughout their business strategy and daily operations to remain relevant to the market ${ }^{[15],[16]}$.

Natural Language Processing (NLP) applications are harness insights from large stores of data from claims and customer support tickets. And it can help underwriters pull up the relevant data on the risks they are writing using search-based analytics to speed up data access ${ }^{[17]}$.

Artificial Intelligence (AI) and Machine Learning (ML) based data models will be widely used to analyze the real-time insurance data for precise risk classification and innovation etc. Insurance companies will have a unique opportunity to leverage AI to foster better customer relationships, user experience, cost optimization, market competitiveness and operational efficiencies ${ }^{[18]}$.

\section{APPROACHES TO SUCCESFUL DIGITAL TRANSFORMATION}

Digital transformation will create challenges for insurance companies in terms of data storage, safety and privacy rules like General Data Protection Regulation (GDPR) in Europe and requires methods to support organizations in their change process. And to start digital transformation journey, companies need assessment of their maturity levels of organizational capabilities and based on such assessment they need to take baby steps ${ }^{[19], ~[20], ~[21] . ~}$

\subsection{Identify What Generates Maximum Value by Using 80:20}

Implementing the digital transformation for insurance is more challenging as compared with other industries such as retail, e-commerce or banking. This is primarily due to the nature of business where multiple stakeholders with competing priorities are involved such as regulators, brokers, policyholders, and shareholders. While regulators' job is to ensure that adequate reserves and cover is provided to the policyholders, policyholders would want to gain maximum coverage on lower premiums; and shareholders would expect maximum Return on Investment (ROI).

Due to the nature of competing priorities within insurance business, the most important work is to prioritize what delivers maximum value for end customers. In the 80:20 approach, the digital transformation team must identify which items can deliver $80 \%$ results for end customers with $20 \%$ efforts. This guiding principle helps the transformation team continuously push for north without being pulled south due to pressure from within.

\subsection{Find out How the Problem Can be Solved}

Every problem may be caused due to certain unique circumstances \& challenges. As long as those are understood and broken down into several smaller problem statements, solving the larger problem becomes easier. Once the problems are identified, next step becomes finding the best solution which can fix those problems or mitigate their impacts.

Finding a solution can be a multifaceted approach from identifying right technical and non-technical resources which can solve the problem. Exploring always for new solutions that work for the given case may be a good practice; however, most of the time it's important to get something implemented and released for customers rather than waiting for the best solution.

\subsection{Execution Phase - Do It Agile \& Let the Market Validate It}

As soon as top value generating digital transformation priorities are identified, the execution phase must start, and 
insurance companies should adopt Agile implementation. Doing digital transformation in an agile way gives companies an opportunity to build a workable solution for the end customers from the market and receive the continuous feedback whether transformation works.

The agile setup creates self-sufficient teams which organizes and manages itself to deliver a workable solution for business end customers. Agile implementations require changes in the organization culture, and it must be part of the corporate strategy to successfully transform into an agile organization to ensure that planned value is delivered.

\subsection{Fail Fast, Learn \& Repeat}

Iteration \& having the opportunity to implement the learning from the market is the best way to validate transformation assumptions and incorporate the changes needed in the entire digital transformation landscape. In many cases, the hypothesis would fail completely, and time must be invested to redo things; however, it's extremely important to know sooner rather than later that hypothesis is failed.

Every failed hypothesis must be translated into certain learning which must lead to new ways of doing things until it gets right. Digital transformation always poses unknown challenges as organizations are charting out into new technological and cultural territories; so, failing fast, learning from those failures and rebuilding becomes more important to ensure that transformation delivers the maximum and desired values for organization end customers.

\section{SUMMARY AND CONCLUSIONS}

Digital transformation equipped with artificial intelligence (AI) is going to provides a wealth of new opportunities. As per a survey conducted by Forrester, executives predict digital will influence $47 \%$ of total sales by $2020^{[22]}$.

You can't transform everything in one go, so constant focus and drive is required to identifying new ways to redefine the way an organization should work in the new digital era. There's no single pill for digital transformation and it requires a long-term leadership vision for changes to happen across organizations. Focus, courage and culture to adapt digital and innovative ways is very crucial aspect of this transformations [23], [24], [25]

The biggest advantage of adopting digital transformation is that as more and more data is collected and analyzed, the AI systems gets better at providing accurate results and speeds-up the overall insurance process. And key to digital transformation is constant focus on changing customer requirements, flexibility, technology awareness and adaptability to the incremental changes etc ${ }^{[26],[27]}$.

\section{ACKNOWLEDGEMENTS}

Our thanks to the experts who have contributed towards development of the template

\section{REFERENCES}

[1] How Digital Transformation is Changing the Insurance Industry, https://global.hitachisolutions.com/blog/insurance-digital-transformation

[2] $\mathrm{AI}$ and CSP: Keys to digital transformation in insurance,https://www.propertycasualty360.com/2020/05 /25/ai-and-csp-keys-to-digital-transformation-ininsurance/
[3] The power of disruption: Digital transformation in insurance,https://www.propertycasualty360.com/2020/03 /20/the-power-of-disruption-digital-transformation-ininsurance/?slreturn=20200725111354

[4] Disrupt or Die: The Emerging Role of AI Technologies in the Insurance Market, https://www.exlservice.com/disrupt-or-die-the-emergingrole-of-ai-technologies-in-the-insurance-market

[5] Embracing Digital Is The Key To An Organization's Future Success, https://www.vlinkinfo.com/embracingdigital-is-key-to-success/

[6] Why it's important to embrace digital technology, https://www.unily.com/insights/blogs/why-it-simportant-to-embrace-digital-technology

[7] Porter's Value Chain- Understanding How Value Is Created Within Organizations, https://www.mindtools.com/pages/article/newSTR_66.ht $\mathrm{m}$

[8] The value chain approach to insurance, https://www.captiveinternational.com/contributedarticle/the-value-chain-approach-to-insurance

[9] Reinforcing the Insurance Value Chain, https://www.verisk.com/verisk-review/winter2016/reinforcing-the-insurance-value-chain/

[10] The value chain approach to insurance, https://www.captiveinternational.com/contributedarticle/the-value-chain-approach-to-insurance

[11] 3 ways AI is transforming the insurance industry, https://thenextweb.com/growth-quarters/2020/02/24/3ways-ai-is-transforming-the-insurance-industry/

[12] Insurance 2030-The impact of AI on the future of insurance,https://www.mckinsey.com/industries/financial -services/our-insights/insurance-2030-the-impact-of-aion-the-future-of-insurance

[13] AI in insurance communication offers insurers a competitive edge, https://www.striata.com/how-aitechnology-will-change-the-future-of-insurance/

[14] Three ways AI will transform the insurance industry, https://www.ey.com/en_in/insurance/three-ways-ai-willtransform-the-insurance-industry

[15] Top 5 ways AI is transforming the insurance industry, https://www.act21.io/top-5-ways-ai-is-transforming-theinsurance-industry/

[16] How Insurance Companies are Using Artificial Intelligence to Transform an Industry, https://innodata.com/insurance-data-annotation/

[17] $\mathrm{AI}-$ the new magic in insurance, https://www.expresscomputer.in/columns/ai-the-newmagic-in-insurance/57653/

[18] How can insurers realise the true value of AI? https://www.fintechmagazine.com/insurance/how-caninsurers-realise-true-value-ai

[19] Five major challenges of insurance digital transformation, https://www.propertycasualty360.com/2020/08/26/fivemajor-challenges-of-insurance-digital- 
transformation/?slreturn $=20200726085248$

[20] The Nine Elements of Digital Transformation, https://sloanreview.mit.edu/article/the-nine-elements-ofdigital-transformation/

[21] Digital transformation: online guide to digital business transformation, https://www.i-scoop.eu/digitaltransformation/

[22] Digital continues to disrupt, https://www.forrester.com/staticassets/marketing/blogs/F orresterInfographicDigitalBusinessNigelFenwick18.pdf

[23] World Economic Forum WhitePaper- Digital Transformation of Industries: In collaboration with Accenture, http://reports.weforum.org/digitaltransformation/wpcontent/blogs.dir/94/mp/files/pages/files/digitalenterprise-narrative-final-january-2016.pdf
[24] Digital Doesn't Have to Be Disruptive, https://hbr.org/2019/07/digital-doesnt-have-to-bedisruptive

[25] Embracing the Digital Era- What We Need Is a Vision, a Structured Approach and Experimentation, https://www.compact.nl/en/articles/embracing-thedigital-era/

[26] Unlocking success in digital transformations, https://www.mckinsey.com/business-

functions/organization/our-insights/unlocking-successin-digital-transformations

[27] Business $4.0^{\mathrm{TM}}$ - The behaviors of digital transformation, https://www.tcs.com/content/dam/tcs/pdf/discovertcs/business/The\%20Behaviors\%20of\%20Digital\%20Tra nsformation.pdf 